\title{
Prediction of Newhall navel orange internal quality based on digital microscopy
}

\author{
Shumin $\mathrm{Gao}^{1,2,6 \dagger}$, Cong $\mathrm{CaO}^{2,4,6 \dagger}$, Dingyi $\mathrm{Hu}^{1,2,6}$, Rangwei $\mathrm{Xu}^{3,5}$, Yunjiang Cheng ${ }^{3,5}$, \\ Hong Chen ${ }^{1,2,6}$, Ming Zhu ${ }^{1,2}$, Shanjun $\mathrm{Li}^{1,2,3,6 *}$ \\ (1. College of Engineering, Huazhong Agricultural University, Wuhan 430070, China; \\ 2. Key Laboratory of Agricultural Equipment in Mid-lower Yangtze River, Ministry of Agriculture and Rural Affairs, Wuhan 430070, China; \\ 3. National R\&D Center for Citrus Preservation, Huazhong Agricultural University, Wuhan 430070, China; \\ 4. Nanfang College of Sun Yat-sen University, Guangzhou 510970, China; \\ 5. College of horticulture \& forestry sciences, Huazhong Agricultural University, Wuhan 430070, China; \\ 6. Citrus Mechanization Research Base, Ministry of Agriculture and Rural Affairs, Huazhong Agricultural University, \\ Wuhan 430070, China)
}

\begin{abstract}
The traditional technology for internal quality detection of citrus fruit is believed to be destructive, inefficient, and error-prone. To address these issues, a novel method has been developed to evaluate nondestructively the sugar-acid ratio (SAR) of soluble solids by using the oil glands density (OGD). In this study, a total of 584 samples with different widths were collected. The sample data were correlated that the SAR and OGD decreased with time during storage. The relevance with time was significant between SAR and OGD of citrus positively correlated in the calibration group $\left(R^{2}=0.82\right)$, which indicated that OGD could be used to predict the internal quality of citrus. It indicated that a new generation of digital microscopy could provide an alternative to predict nondestructively the internal quality of citrus fruit, as well as some theoretical insight for further studies of online quality detection in the future.
\end{abstract}

Keywords: citrus, nondestructive, oil glands, internal quality, storage, microscopy

DOI: $10.25165 /$ j.ijabe.20211406.5996

Citation: Gao S M, Cao C, Hu D Y, Xu R W, Cheng Y J, Chen H, et al. Prediction of Newhall navel orange internal quality based on digital microscopy. Int J Agric \& Biol Eng, 2021; 14(6): 222-227.

\section{Introduction}

Fruits quality characteristics, including external quality (such as color, size, and shape $)^{[1]}$ and internal quality (such as soluble solids, titratable acid, and firmness) ${ }^{[2]}$, are the basic factors affecting the consumers' desire of purchasing ${ }^{[3,4]}$. Nowadays, consumers' pursuit of fruits quality is not only limited to external quality but also extends to internal quantity. Citrus is planted widely in the world for its rich in vitamins, carotenoids, minerals, and dietary fibers ${ }^{[5-8]}$, which are essential for normal human growth and development and overall nutritional health. Soluble solids content (SSC) is one of the most crucial internal attributes determining consumers' purchasing desire also plays an important role in guiding orchard management. Because sugar contents

Received date: 2020-07-07 Accepted date: 2021-09-01

Biographies: Shumin Gao, $\mathrm{PhD}$, research interest: nondestructive detection of agricultural products, Email: 1049976177@qq.com; Cong Cao, MS, research interest: nondestructive detection of agricultural products, Email: 296696741@qq.com; Dingyi Hu, MS, research interest: nondestructive detection of agricultural products, Email: hya1996@126.com; Rangwei Xu, MS, research interest: preservation, storage and transportation of citrus, Email: xurang001@163.com; Yunjiang Cheng, Professor, research interest: preservation, storage and transportation of citrus, Email: yjcheng@ mail.hzau.edu.cn; Hong Chen, $\mathrm{PhD}$, research interest: nondestructive detection of agricultural products, Email: chenhong@mail.hzau.edu.cn; Ming Zhu, Professor, research interest: agricultural products processing, Email: 13801392760@163.com.

$\uparrow$ These authors contributed equally to this work.

*Corresponding author: Shanjun $\mathbf{L i}, \mathrm{PhD}$, Professor, research interest: nondestructive detection of agricultural products, College of Engineering, Huazhong Agricultural University, Wuhan 430070, China. Tel: +8613071289256, Email: shanjunlee@mail.hzau.edu.cn. generally determine the flavor of fruits. After harvest, the texture of citrus fruit changes constantly, and the flavor deteriorates ${ }^{[9]}$. These factors severely restrict the improvement of citrus economic benefits and its market competitiveness. As the world is changing at a rapid pace, driven by science and technology, the general public has drawn their attention to the issue of food quality and safety ${ }^{[10]}$. Thus detection has performed a key role in improving fruit quality, enhancing fruit market competitiveness, and increasing the income of farmers ${ }^{[11]}$. However, the traditional testing technology of SSC is destructive, time-consuming and high energy consumption, which is not suitable for the demand of fast and large amounts of fruits grading industry ${ }^{[2]}$.

Nondestructive testing technology has the advantages of real-time, low energy consumption and minimal sample preparation. In recent years, it has been extensively studied and accepted as an effective method for the detection of fruits maturity, shelf life, quality sorting, and even internal disease. In general, nondestructive testing technologies include electronic nose ${ }^{[12-14]}$, visible/near-infrared (Vis/NIR) spectroscopy $^{[15-17]}$, ultrasonic sensing technique ${ }^{[18,19]}$, machine vision sensing technique ${ }^{[20,21]}$.

To be frank, the electronic nose is well-known that sample preparation and sampling are error-prone steps for e-nose measurements. Gas sensors are very sensitive to temperature, humidity, pressure, gas velocity, and vapor concentration. $\mathrm{Lyu}^{[22]}$ et al. combined NIR spectrometer with electronic nose technology to detect SSC of Ehime Prefecture Jell-O orange No. 38 more accurately. The $R$ and RMSE of the validation set model were 0.8872 and 0.4709 respectively and the result shows that PCA-BPNN based on the fusion data has better SSC prediction ability. However, sample preparation of e-nose sensing is also 
very challenging since the number of volatiles released from foods depends on many factors such as temperature, pressure, and humidity. High repeatability and precision of e-nose measurement require strict control of sample preparation and sampling environment ${ }^{[23]}$.

Visible and near-infrared reflectance spectroscopy has become a powerful tool for the non-destructive monitoring and prediction of multiple quality and safety attributes of agro-food products ${ }^{[24]}$, Wang et al. ${ }^{[25]}$ had compared three kinds of spectral acquisition methods, diffuse reflection, transmission and diffuse transmission, to realize the detection of SSC in navel orange. The results showed that no matter how the sample is peeled or not, the transmission mode had the best result, and adding visible light region data will reduce the prediction accuracy of the model. However, it is difficult to the high-speed movement of the samples.

Ultrasound is in the wide ranges of frequency and power, easy usage and the safety of this technology for humans, made it an appropriate technique to apply in the different fields of fruits, juices and dairy industries including analyzing, control and processing, Mizrach has developed a non-destructive ultrasonic testing system, which used a set of low-frequency probes to measure the ultrasonic signals sent and received through the distance for evaluating the maturity and hardness of fruits ${ }^{[26]}$, but researches in the field of ultrasound in the food science are conducted under controlled laboratory conditions, and it cannot be expected that the same obtained experimental results would be accessible on an industrial scale $^{[27]}$.

The advantage of machine vision is that provides rapidness, persistence, and non-destructiveness ${ }^{[28]}$.

Above all, the method of machine vision was used to observe citrus epidermis, secretory cavities occur naturally in all species of $^{[16]}$ the family Rutaceae ${ }^{[29]}$. In the genus citrus, they are commonly referred to as oil glands and occur in the stem, mesophyll of leaves, all parts of the flower except the stamens, and the fruit, where they are positioned in the exocarp or 'flavedo' layer of the rind amongst compact subepidermal parenchyma tissue and Knight found that the thickness of oil gland layer increased and the density of oil gland ${ }^{[30-34]}$ decreased with the fruit enlargement ${ }^{[33]}$. Guo et al. ${ }^{[35]}$ measured the content of citrus peel essential oil under different storage conditions and showed that the content of citrus peel essential oil decreased with the increase of storage time, based on this, the decrease of essential oil leads to the decrease of oil gland density under machine vision. What is more, little information has been reported on the correlation between oil glands density (OGD) in the citrus epidermis and citrus internal quality. After the experiment in the study, there is a certain statistical law between the density of citrus oil gland and SSC.

In this study, a new nondestructive detection method, namely, continuous detection of the changes in oil glands in citrus epidermis to predict the changes in soluble solids in citrus was proposed. This method can be used to predict the internal quality of citrus by the morphological changes in oil glands. With further related research, this method will be part of a potential rapid online detection method.

\section{Materials and methods}

\subsection{Sample preparation}

Newhall navel orange (Citrus synesis) samples were collected from 15-year-old commercial fruit orchards in Gigue County, Hubei Province, China. Fruits with diameters of $80 \mathrm{~mm}$ and above (A), $75 \mathrm{~mm}$ to $80 \mathrm{~mm}$ (B), $70 \mathrm{~mm}$ to $75 \mathrm{~mm}$ (C), and $65 \mathrm{~mm}$ to $70 \mathrm{~mm}(\mathrm{D})$ were picked when they reached commercial maturity in early December. On the same day, the fruits were transported back to Huazhong Agricultural University, Wuhan, Hubei, China. The fruits were graded based on the four diameter ranges, and fruits with deformities and surface defects were eliminated. After a thorough cleaning with clean water, the fruits were air-dried. After $24 \mathrm{~h}$ of air-drying process, the fruits were packed in polyethylene bags with a thickness of $0.01 \mathrm{~mm}$ and stored at room temperature in a ventilated warehouse. The storage temperature was $12^{\circ} \mathrm{C}-20^{\circ} \mathrm{C}$, and the relative humidity was $80 \%-85 \%$. After $24 \mathrm{~h}$ storage, sampling, observation and detection were conducted for the first time and then every $10 \mathrm{~d}$ for an additional 6 times. The specific sampling methods were as follows: Twenty fruits were selected from each of the four diameter ranges. Eight circular areas with a diameter of $1.5 \mathrm{~cm}$ were selected along the equator of each fruit to detect the oil glands changes. A total of 584 samples were selected with 504 (63 samples from each category of four different diameters) subjected to respiratory intensity (252 samples) detection and soluble solid (252 samples) detection, while another 80 samples were used to detect the density of oil glands in the epidermis. The detection was performed for every 10-d interval.

\subsection{VHX-6000 imaging system and measurement method}

The system of VHX-6000 (shown in Figure 1) consisted of a highly sensitive, high-speed 50-frame CMOS camera, TRIPLE'R wireless RZ lens, small high-speed motor, high-resolution plus high-speed XYZ electric platform, and REMAX VI imaging software. SPSS 17.0 and Origin 9.0 software were used for data processing. The Optoelectronic 3D color microscope could automatically measure areas, count oil glands, and calibrate the photographed images with an automatic elimination of man-made errors. During the detection, VH-Z20R/Z20T super miniature high-performance zoom lenses were used with a stable light source and the observation distance was $25.5 \mathrm{~mm}$.

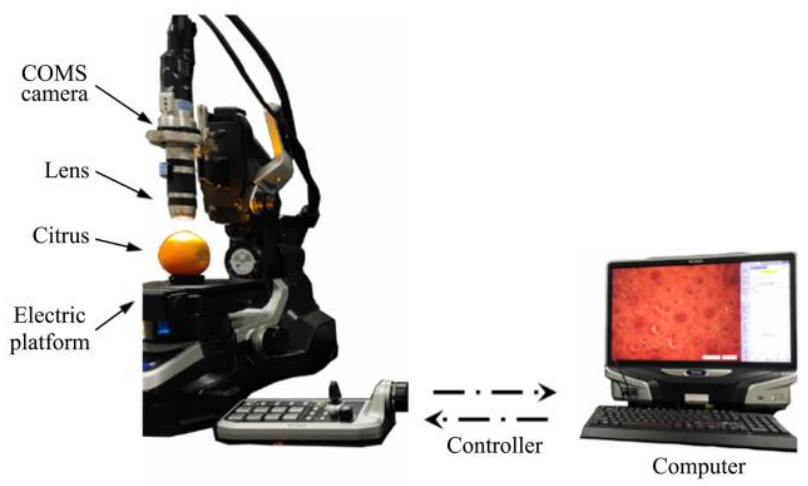

Figure 1 VHX-6000 imaging system

The flow chart of nondestructively predicting the internal quality of citrus fruit is shown in Figure 2. During the storage of citrus, the essential oils in the oil glands were absorbed or volatilized, which caused some characteristics of the oil glands to change. According to the experimental data, analyze the correlation between sugar-acid ratio (SAR) and OGD.

\subsection{Determination of physical and chemical indexes}

The basic morphological parameters and quality of navel orange samples were measured. Citruses with four different diameters were measured by Deli high precision electronic digital Vernier caliper (model DL91150, Deli Group Ltd., China). The sample fruits with different diameters (equatorial circumference) were weighed using a $1 \%$ electronic balance scale (model MP31001, Shanghai Hanging Scientific Instrument Co., Ltd., China). The content of total soluble solids (TSS) was determined 
by PAL-1 (pocket refractometer PAL-1, Atagi, Japan). The content of titratable acid (TA) was determined by acidity meter according to the manufacturer's instructions ${ }^{[36]}$. Nine fruits of the same size were combined into one group, and three fruits from the same group were squeezed into juice together. Then, $0.3 \mathrm{~mL}$ of the juice was added to $30 \mathrm{~mL}$ of distilled water. After blending, the TA content was determined using a digital acidity meter (GMK-835, G-WON HITECH Co., Ltd., Korea) ${ }^{[37]}$.

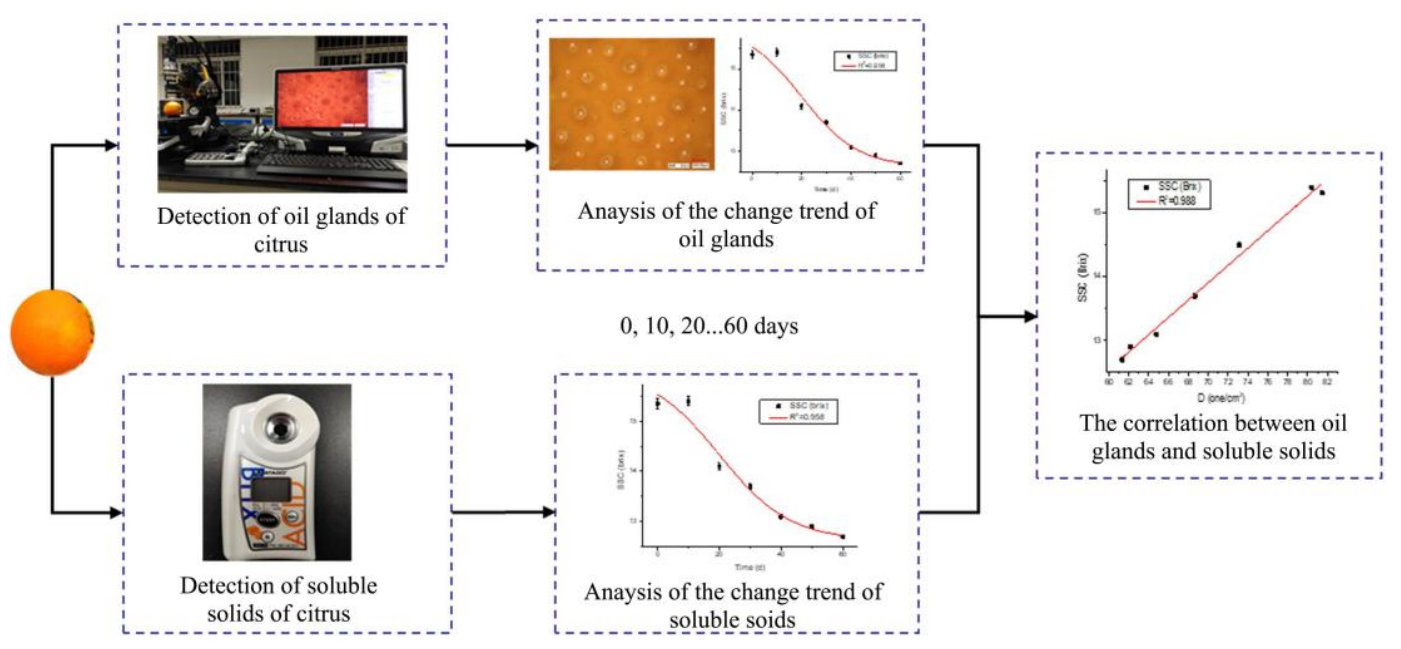

Figure 2 Flow chart of nondestructively predicting internal quality of citrus experiment

Three fruits from one group were selected and weighed, and this activity was repeated three times. A total of 36 fruits from 4 groups were sealed in a $2.6 \mathrm{~L}$ box for maintaining freshness and placed at room temperature for $2 \mathrm{~h}$. Gas was extracted from the sealed box with a $1 \mathrm{~mL}$ syringe. The concentration of $\mathrm{CO}_{2}$ in the $1 \mathrm{~mL}$ of the extracted gas was determined by gas chromatography (Agilent 7890A, USA) ${ }^{[37]}$.

\section{Results and discussion}

This study found that the number of oil glands decreased gradually with the prolongation of storage time ${ }^{[37]}$, as shown in

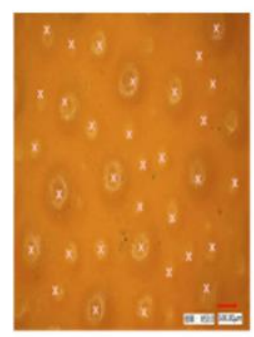

a. $0 \mathrm{~d}$,

number $=33 \pm 5$

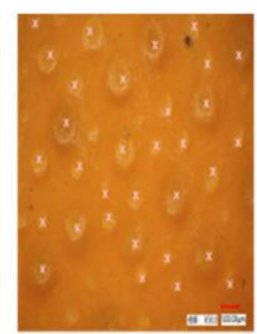

b. $10 \mathrm{~d}$, number $=32 \pm 4$

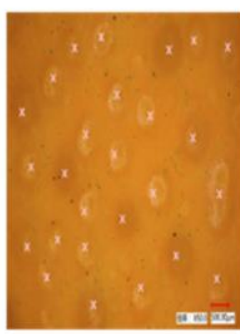

c. $20 \mathrm{~d}$, number $=27 \pm 3$

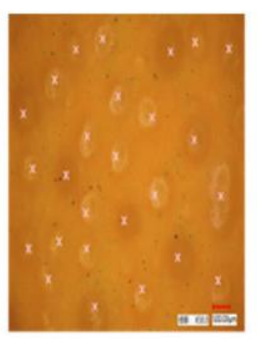

d. $30 \mathrm{~d}$, number $=26 \pm 3$
Figure 3. It was observed that the number of oil glands was (35 \pm 5$)$ at $0 \mathrm{~d},(33 \pm 4)$ at $10 \mathrm{~d},(27 \pm 3)$ at $20 \mathrm{~d},(26 \pm 3)$ at $30 \mathrm{~d},(24 \pm 3)$ at $40 \mathrm{~d}$, $(23 \pm 2)$ at $50 \mathrm{~d}$, and $(21 \pm 2)$ at $60 \mathrm{~d}$, and the detection area was $3.78 \times 10^{-5} \mathrm{~m}^{2}$. The analysis showed that the decreasing trend of oil glands was obvious within day 10 to day 20 and that the changing trend of oil glands slowed down from $20 \mathrm{~d}$ to $60 \mathrm{~d}$. At the beginning of storage, the OGD per unit area of citrus was high. With the prolongation of storage time, the number of oil glands and the size of oil glands decreased gradually. Therefore, a parameter was introduced to describe the change in OGD in the epidermis of citrus during storage.

Figure 3 Example images of oil glands number changes with storage time

Figure 3 shows that the oil glands of fresh citrus epidermis exhibited obvious characteristics. With increasing storage time, the number of oil glands gradually decreased respiration. The OGD was calculated as follows:

$$
\text { OGD }=\frac{\text { Number }}{\text { Area }}
$$

where, the number of oil glands referred to the total number of oil glands in the detection area, and the area was the projected area of the detection area $\left(3.78 \times 10^{-5} \mathrm{~m}^{2}\right)$. OGD indicates the number of oil glands per unit area, $\mathrm{m}^{-2}$.

The detection area was divided into eight equal circular areas with a diameter of $1.5 \mathrm{~cm}$ distributed evenly on the equator of each citrus fruit.

The results show that the SAR of citrus also exhibited a decreasing trend with increasing storage time. As shown in Figure 4, the SAR increased temporarily within $0 \mathrm{~d}$ to $10 \mathrm{~d}$, which might be explained by the fact that after the citrus was picked from the tree, the acid content in the citrus decreased at a faster speed than the sugar content in the citrus in the early storage period. However, the SAR significantly decreased rapidly within $10 \mathrm{~d}$ to $20 \mathrm{~d}$. During $20 \mathrm{~d}$ to $60 \mathrm{~d}$, the decrease in the SAR gradually slowed down, which was similar to the trend in the OGD shown in Figure 4a. Based on the observed similar trends, the correlation between OGD and SAR was analyzed to establish a model for describing the internal relationship between these two parameters.

The correlation between SAR and OGD of the four fruit diameters is shown in Figure 5. The correlation $R^{2}$ of fruit diameters A, B, C and D is 0.733, 0.765, 0.791 and 0.937, respectively.

It can be seen from Figure 5 that the size of fruit diameter has a negative correlation with the correlation between SAR and OGD. The smaller the fruit diameter is, the higher the correlation between SAR and OGD. 


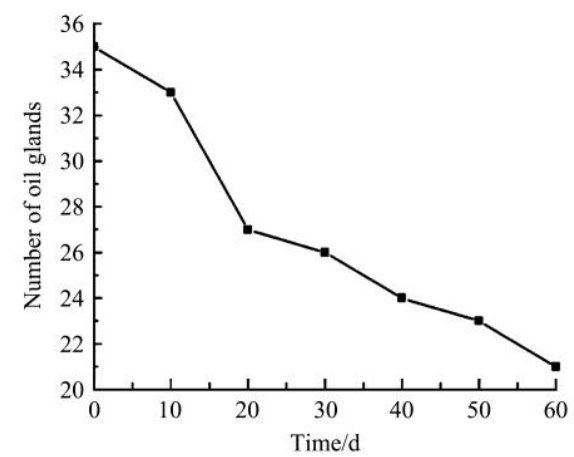

a. Variation trend of average number of oil glands

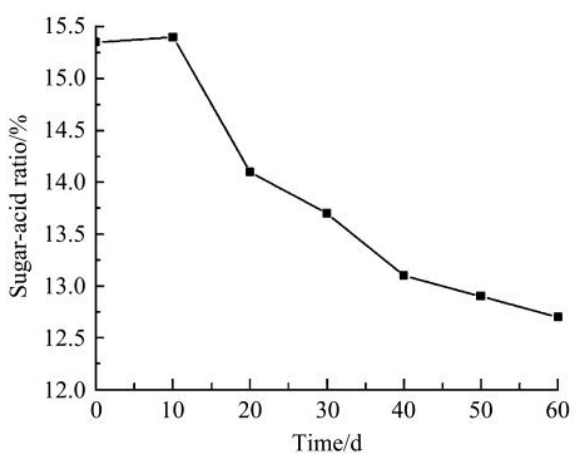

b. Change trend of average sugar-acid ratio

Figure 4 Chart showing trends for the number of oil glands and SAR

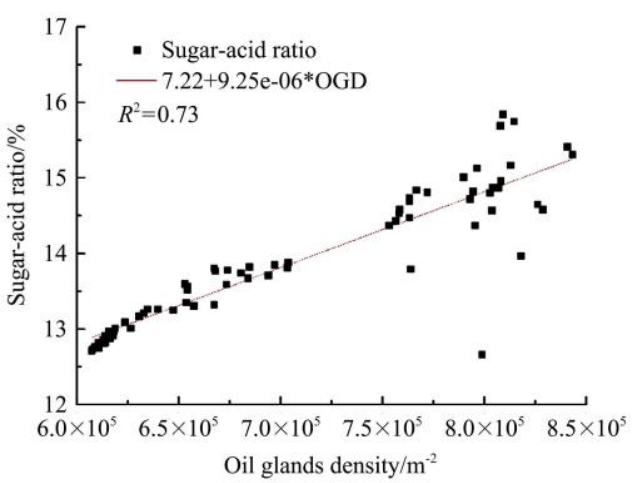

a. Fruit diameters $\geq 80 \mathrm{~mm}$

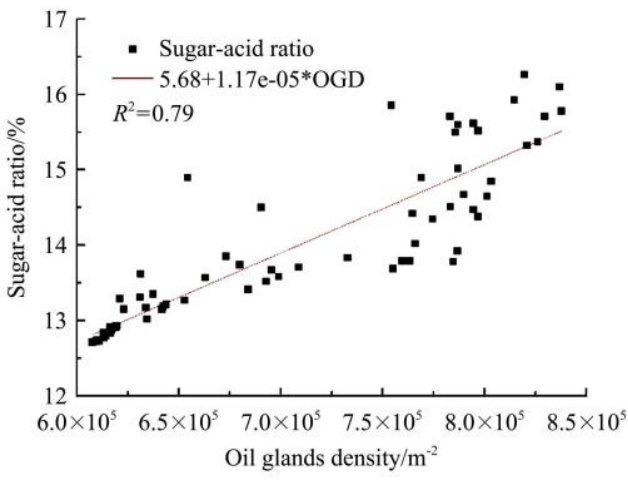

c. Fruit diameters of $70-75 \mathrm{~mm}$

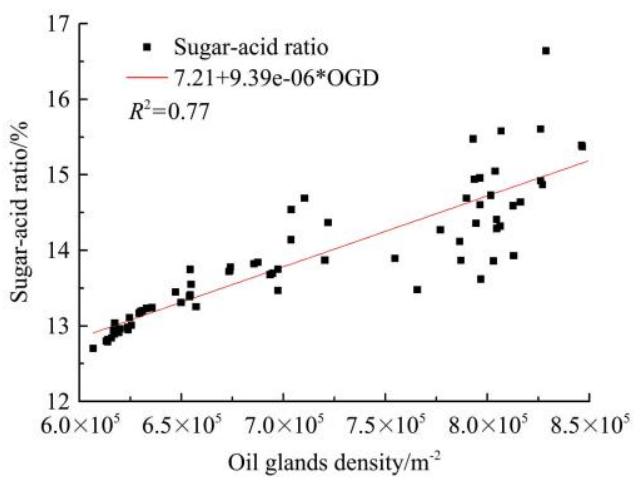

b. Fruit diameters of $75-80 \mathrm{~mm}$

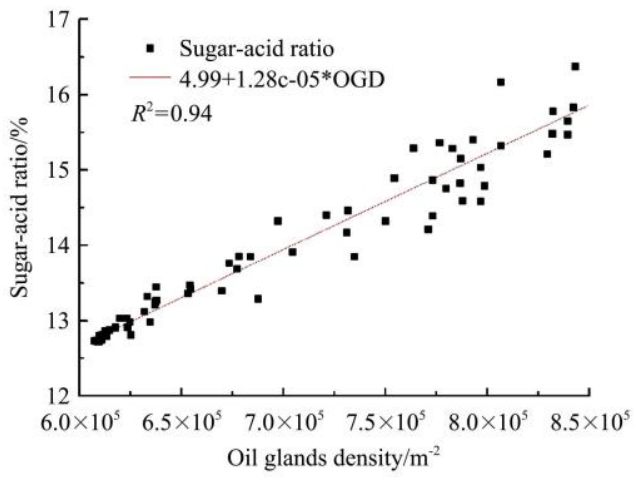

d. Fruit diameters of $65-70 \mathrm{~mm}$

Figure 5 Correlation fitting curve of SAR and OGD for four diameters of navel orange

Citrus is a non-climacteric fruit, as can be seen from Figure 6. Although respiration decreased, the consumption of essential oil by oil glands was lower than expected within $0 \mathrm{~d}$ to $10 \mathrm{~d}$ of storage. Therefore, there was no significant change of OGD in $0 \mathrm{~d}$ to $10 \mathrm{~d}$ of storage. From $10 \mathrm{~d}$ to $40 \mathrm{~d}$, the respiratory intensity decreased rapidly. However, the respiratory intensity remained at a relatively high level compared with that in the late period of storage (40-60 d). Affected by the change in respiratory intensity, the OGD decreased gradually. With the increase in storage time (from $10 \mathrm{~d}$ to $40 \mathrm{~d}$ ), the small oil glands gradually disappeared and the OGD obviously decreased. From $40 \mathrm{~d}$ to $60 \mathrm{~d}$, the decrease in OGD gradually slowed down, which might be attributed to the fact of slowly decreasing in respiratory intensity. Then the activity of oil glands in the citrus epidermis decreased, which resulted in the decrease in OGD was gradually slowed.

The physical parameters of citrus fruits, i.e., the OGD and SAR, are listed in Table 1. The OGD and SAR were measured in the citrus samples of the calibration group.

Figure 7 shows the fitting curves of SAR and OGD of all samples, and it is obvious that they have a strong correlation. Correlation coefficient $R^{2}$ is 0.82 .
The fitting curve equation of the OGD and SAR was as follows:

$$
\text { SAR }=6.20114+\left(1.09307 \times 10^{-5}\right) \text { OGD }
$$

where, SAR is the calibrated sugar-acid ratio, \%; OGD is the density of oil glands (number of oil glands per unit area), $\mathrm{m}^{-2}$.

The correlation analysis results showed that the OGD was highly positively correlated with the internal quality with an $R^{2}=0.82$.

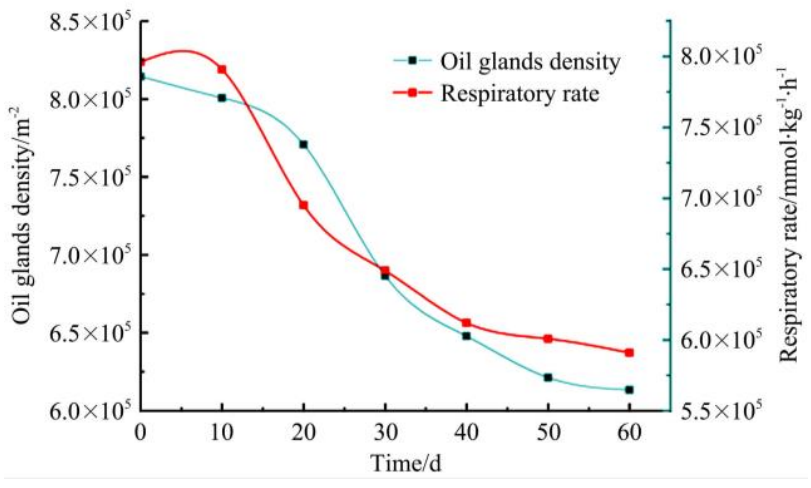

Figure 6 Diagram of respiratory rate, OGD of the calibration group 
Table 1 OGD and SAR physicochemical parameters of navel orange samples in the calibration group

\begin{tabular}{|c|c|c|c|c|c|c|}
\hline \multirow{2}{*}{ Time/d } & \multicolumn{2}{|c|}{ Range } & \multicolumn{2}{|c|}{ Mean } & \multicolumn{2}{|c|}{ Standard deviation } \\
\hline & $\mathrm{OGD} / \mathrm{m}^{-2}$ & SAR & $\mathrm{OGD} / \mathrm{m}^{-2}$ & SAR & $\mathrm{OGD} / \mathrm{m}^{-2}$ & SAR \\
\hline 0 & 784507-859105 & $13.78-15.71$ & 787205.89 & 15.54 & 19573.00 & 0.85 \\
\hline 10 & 763284-846108 & $13.79-15.39$ & 779568.72 & 15.37 & 26220.26 & 0.58 \\
\hline 20 & $754958-803785$ & $13.69-15.05$ & 743705.61 & 15.00 & 22231.73 & 0.55 \\
\hline 30 & $699067-703806$ & $13.58-14.54$ & 653754.89 & 14.00 & 17563.71 & 0.36 \\
\hline 40 & $641798-685623$ & $13.15-13.82$ & 614012.94 & 13.38 & 18038.94 & 0.25 \\
\hline 50 & $617260-632891$ & $12.89-13.23$ & 588541.22 & 13.03 & 8530.17 & 0.16 \\
\hline 60 & $609732-615817$ & $12.73-12.84$ & 579257.94 & 12.81 & 5259.54 & 0.09 \\
\hline
\end{tabular}

Note: OGD represents the oil glands density; SAR represents the sugar-acid ratio.

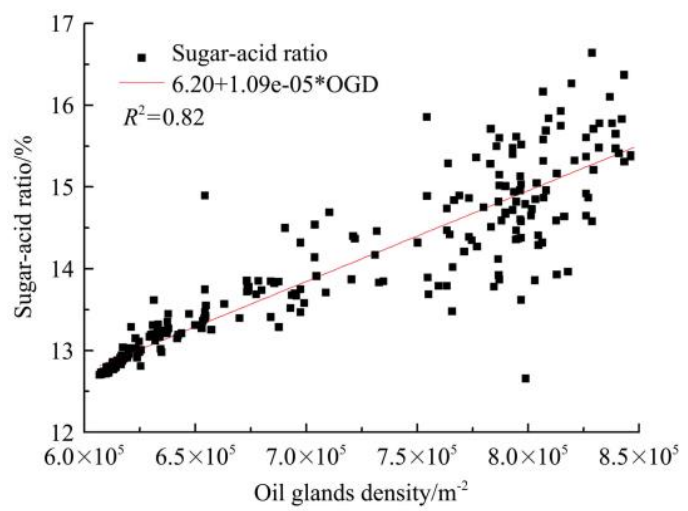

Figure 7 Correction group SAR and OGD fitting curve

\section{Conclusions}

Overall, the correlation analysis results showed that the SAR in the citrus was highly positively correlated with the OGD in both the calibration group $\left(R^{2}=0.82\right)$, which indicated that the OGD could be used to predict the internal quality of citrus. Further research can establish more accurate models for different fruit diameters and explore corresponding models for different varieties. In addition, this study developed a new digital microscopy-based method for nondestructively predicting the internal quality of citrus fruit. It also provides a theoretical basis for further investigation of the nondestructive detection of citrus quality.

\section{Acknowledgements}

This work was financially supported in part by the National Key R\&D Projects of China (Grant No. 2017YFD0701405), and the Earmarked Fund for Modern Agro-industry (Citrus) Technology Research System of China (Grant No. CARS-27), and both Special Funds for the Construction of Industrial Technology System of Modern Agriculture (Citrus) (CARS-26) and National Key R\&D Program (Grant No. 2020YFD01000101), the Construction Project of Citrus Whole Course Mechanized Scientific Research Base (Agricultural Development Facility [2017]19), Hubei Agricultural Science and Technology Innovation Action Project.

\section{[References]}

[1] Lembe S M, Umezuruike, L O, Paul C, Sandra L, Hélène H N, Abdul M, et al. Assessment of rind quality of 'Nules Clementine' mandarin fruit during postharvest storage: 2. Robust Vis/NIRS PLS models for prediction of physico-chemical attributes. Scientia Horticulturae, 2014; 165: 421-432.

[2] Tian X, Li J B, Yi S L, Jin G Q, Qiu X Y, Li Y J. Nondestructive determining the soluble solids content of citrus using near infrared transmittance technology combined with the variable selection algorithm. Artificial Intelligence in Agriculture, 2020; 4: 48-57.

[3] Baldwin E A, Bai J, Plotto A, Ritenour M A. Citrus fruit quality assessment; producer and consumer perspectives. Stewart Postharvest Review, 2014; 10(2): 1-7.

[4] Dan C, Şerban C, Sestras A F, Militaru M, Morariu P, Sestras R E. Consumer perception concerning apple fruit quality, depending on cultivars and hedonic scale of evaluation - a case study. Notulae Scientia Biologicae, 2015; 7(1): 140-149.

[5] Ghanem N, Mihoubi D, Kechaou N, Mihoubi N B. Microwave dehydration of three citrus peel cultivars: Effect on water and oil retention capacities, color, shrinkage and total phenols content. Ind Crops Prod, 2012; 40(1): 167-177.

[6] Mahato N, Sharma K, Sinha M, Cho M H. Citrus waste derived nutra-/pharmaceuticals for health benefits: Current trends and future perspectives. J. Funct. Foods, 2017; 40: 307-316.

[7] Nekvapil F, Brezestean I, Barchewitz D, Glamuzina B, Chiş V, Pinzaru S C. Citrus fruits freshness assessment using Raman spectroscopy. Food Chem, 2018; 242: 560-567.

[8] Singh E, Kumari J, Jain P, Sharma S. Nutritional evaluation of dried peel of citrous species and its application in cake development. J Nutr Ecol \& Food Res, 2014; 2(1): 10-14.

[9] Porat R, Deterre S, Giampaoli P, Plotto A. The flavor of citrus fruit Wiley Online Library, 2016; 31p.

[10] Zhao S, Zhang B B, Li J H. On China's policy and countermeasures of quality and safety of farm products and foodstuffs. Food Sci, 2006; 10: 630-635.

[11] Schimmenti E, Borsellino V, Galati A. Growth of citrus production among the Euro-Mediterranean countries: Political implications and empirical findings. Span J Agric Res, 2013; 11(3): 561-577.

[12] Jia W S, Liang G, Tian H, Sun J, Wan C H. Electronic nose-based technique for rapid detection and recognition of moldy apples. Food Chem, 2019; 19(7): 1526. doi: 10.20944/preprints201903.0008.v1.

[13] Li C, Heinemann P, Sherry R. Neural network and Bayesian network fusion models to fuse electronic nose and surface acoustic wave sensor data for apple defect detection. Sens. Actuators B Chem, 2007; 125(1): 301-310.

[14] Ren Y, Ramaswamy H S, Li Y, Yuan C, Reny X. Classification of impact injury of apples using electronic nose coupled with multivariate statistical analyses. J Food Process Eng, 2017; 41(5): e12698. doi: 10.1111/jfpe. 12698 .

[15] Choi J H, Chen P A, Lee B H, Yim S H, Kim M S, Bae Y S, et al Portable, non-destructive tester integrating VIS/NIR reflectance spectroscopy for the detection of sugar content in Asian pears. Sci Hortic, 2017; 220: 147-153.

[16] Oliveira-Folador G, Bicudo M, Andrade E D, Renard M, Bureau S, Castilhos F D. Quality traits prediction of the passion fruit pulp using NIR and MIR spectroscopy. LWT, 2018; 95: 172-178.

[17] Pérez-Marín D, Torres I, Entrenas J A, Vega M, Sánchez M. Pre-harvest screening on-vine of spinach quality and safety using NIRS technology. Spectrochim Acta A, 2018; 207: 242-250.

[18] Aboudaoud I, Faiz B, Aassif E, Moudden A, Izbaim D, Abassi D, et al. The maturity characterization of orange fruit by using high frequency ultrasonic echo pulse method. IOP Conference Series: Materials Science and Engineering, UCIP 2012, Madrid, Spain, 2012; 42: 2038-2042.

[19] Haydar V S, Mohammad G P, Davood M, Ahmad S. Ultrasonic based determination of apple quality as a nondestructive technology. Sensing \& Bio Sensing Research, 2018; 21:22-26.

[20] Guzmán E, Baeten V, Pierna J, García-Mesa J. Determination of the olive maturity index of intact fruits using image analysis. J. Food Sci, 2015; 52(3): 1462-1470.

[21] Kondo N, Ahmad U, Monta M, Murase H. Machine vision based quality evaluation of Iyokan orange fruit using neural networks. Comput Electron Agric, 2000; 29(1-2): 135-147.

[22] Lyu Q, Liao Q, Liu Y, Lan Y. Feasibility of SSC prediction for navel orange based on origin recognition using NIR spectroscopy. IASC, 2015; 21(3): 305-317.

[23] Ilia K, Victor S, Igor K, Ilona K, Ruslan A, Jamila S, et al. On the temporal stability of analyte recognition with an e-nose based on a metal oxide sensor array in practical applications. Sensors, 2018; 18(2): 550. doi: $10.3390 / \mathrm{s} 18020550$.

[24] Cortés V, Blasco J, Aleixos N, Cubero S, Talens P. Monitoring strategies for quality control of agricultural products using visible and near-infrared spectroscopy: A review. Trends Food Sci Technol, 2019; 85: 138-148.

[25] Wang A, Hu D, Xie L. Comparison of detection modes in terms of the necessity of visible region (VIS) and influence of the peel on soluble solids 
content (SSC) determination of navel orange using VIS-SWNIR spectroscopy. J Food Eng, 2014; 126: 126-132.

[26] Mizrach A. Determination of avocado and mango fruit properties by ultrasonic technique. Ultrasonics, 2000; 38(1-8): 717-722.

[27] Firouz M S, Farahmandi A, Hosseinpour S. Recent advances in ultrasound application as a novel technique in analysis, processing and quality control of fruits, juices and dairy products industries: A review. Ultrason Sonochem, 2019; 57: 73-88.

[28] Tripathi M K, Maktedar D D. A role of computer vision in fruits and vegetables among various horticulture products of agriculture fields: A survey. IPA, 2020; 7(2): 183-203.

[29] Fahn A. Secretory tissues in plants: Secretory tissues in plants. Berlin: Springer-Verlag, 1979; 468p.

[30] Bain J M. Morphological, anatomical, and physiological changes in the developing fruit of the Valencia orange, Citrus sinensis (L) Osbeck. Austrilian Journal of Botany, 1958; 6(1): 1-23.

[31] Banafsheh N, Homa R. Structural \& phytochemical indentification on developing processes of oil gland in fruit of bakrai (Citrus reticulata'citrus limetta). Iran: Bakrai mandarin x limetta hybrid, 2009; 365p.

[32] Holtzhausen L C. Observations on the developing fruit of Citrus sinensis cultivar Washington Naval from anthesis to ripeness. Repub S Afr Dep
Agr Tech Serv Tech Commun, South Africa: Pretoria: Govt. Printer, 1969; $15 \mathrm{p}$.

[33] Knight T G, Klieber A, Sedgley M. The relationship between oil gland and fruit development in Washington Navel Orange (Citrus sinensis L. Osbeck). Annals of Botany, 2001; 88(6): 1039-1047.

[34] Zhou X, Yue J, Yang H, Zhu C, Cheng Y. Integration of metabolome, histochemistry and transcriptome analysis provides insights into lignin accumulation in oleocellosis-damaged flavedo of citrus fruit. Postharvest Biol Tec, 2021; 172: 111362. doi: 10.1016/j.postharvbio.2020.111362.

[35] Guo R X, Zhang Y, Tan X H, Wang F, Cai W, Hu X A. Changes of essential oil content in citrus peel during storage at different storage conditions. Food \&\& Machinery, 2012; 28(2): 190-192, 195. (in Chinese)

[36] Obenland D, Collin S, Mackey B, Sievert J, Arpaia M L. Storage temperature and time influences sensory quality of mandarins by altering soluble solids, acidity and aroma volatile composition. Postharvest Biol Tec, 2011; 59(2): 187-193.

[37] Sheng L, Shen D D, Yang W, Zhang M F, Zeng Y L, Xu J, et al. GABA pathway rate-limit citrate degradation in postharvest citrus fruit evidences from HB Pumelo (Citrus grandis) $\times$ Fairchild (Citrus reticulata) hybrid population. J Agric Food Chem, 2017; 65(8): 1669-1676. 